\title{
Current-induced Phase Demodulation Using a PWM Sampling for a Fiber-optic CT
}

\author{
Hyoung-Jun Park \\ Advanced Graduate Education Center of Jeonbuk for Electronics and Information Technology-BK21, \\ Chonbuk National University, Jeonju 561-756, Korea \\ June-Ho Lee \\ Department of Electrical Engineering, Hoseo University, Asan 336-795, Korea \\ Hyun-Jin Kim and Minho Song* \\ Division of Eletronics Engineering, Chonbuk National University, Jeonju 561-756, Korea
}

(Received May 11, 2010 : revised June 23, 2010 : accepted June 24, 2010)

\begin{abstract}
In this work, we used PWM sampling for demodulation of a fiber-optic interferometric current transformer. The interference signal from a fiber-optic CT is sampled with PWM triggers that produce a 90-degree phase difference between two consecutively sampled signals. The current-induced phase is extracted by applying an arctangent demodulation and a phase unwrapping algorithm to the sampled signals. From experiments using the proposed demodulation, we obtained phase measurement accuracy and a linearity error, in AC current measurements, of $\sim 2.35 \mathrm{mrad}$ and $0.18 \%$, respectively. The accuracy of the proposed method was compared with that of a lock-in amplifier demodulation, which showed only $0.36 \%$ difference. To compare the birefringence effects of different fiber-optic sensor coils, a flint glass fiber and a standard single-mode fiber were used under the same conditions. The flint glass fiber coil with a Faraday rotator mirror showed the best performance. Because of the simple hardware structure and signal processing, the proposed demodulation would be suitable for low-cost over-current monitoring in high voltage power systems.
\end{abstract}

Keywords: Fiber-optic CT, Fiber-optic interferometer, Quadrature signal processing, Fiber-optic sensor, Phase extraction

OCIS codes : (060.2370) Fiber optics sensors; (120.5050) Phase measurement; (120.3180) Interferometry; (060.5060) Phase modulation

\section{INTRODUCTION}

Due to increasing demand for electric energy in modern societies, the accurate and credible electric current measurement techniques are required for metering and overcurrent protection of power systems in high-voltage substations. Fiber-optic CTs (current transformers) have many advantages over the conventional conductor winding CTs. These advantages include, EMI (electro-magnetic interference) immunity, the absence of flux saturation and residual flux, high-speed response, low cost in ultra-high-voltage environment, and so on [1-4]. The magnetic field intensity from the electric current generates a phase difference between the two cir- cular polarization modes in the fiber-optic sensor coil. The electric current is evaluated by measuring the phase difference. There are two types of current-induced phase demodulations in the fiber-optic CT systems: polarimetric and interferometric. The undesirable birefringence effect, which originates from the birefringence of the fiber-optic sensor coil, is much smaller in the interferometric scheme than in the polarimetric one. Also the counter-propagating polarization modes in the Sagnac interferometer, which is the most common type of sensor coil in interferometric CTs, travel the identical optical path, thus the effects of environmental perturbations, such as temperature drifts and vibrations, are compensated, making it possible to do high-resolution electric current meas-

\footnotetext{
*Corresponding author: msong@chonbuk.ac.kr

Color versions of one or more of the figures in this paper are available online.
} 
urements [5-7]. The interferometric CTs generate sinusoidal outputs against current variations. Therefore, phase extracting techniques are required to avoid signal fading, limit of phase range, unknown input phase direction, etc [8-10]. Since the working principles are almost the same, the fiber-optic gyroscope signal processing techniques using lock-in amplifiers and digital samplings have been widely used to demodulate the current-induced phase information in the commercial fiberoptic CTs $[6,7]$. In previous research, we used a time-delayed quadrature sampling which we have successfully employed for fiber Bragg grating sensor demodulation [11, 12].

In this work, to demodulate an interferometric fiber-optic CT, we used PWM (pulse width modulation) quadrature sampling. Due to its simple nature, we could obtain a measurement resolution which is good enough for most of the protection uses with much simpler hardware structure and signal processing than for gyroscope demodulation.

\section{PRINCIPLE OF OPERATION}

Figure 1 is the schematic diagram of the proposed interferometric CT that uses a PWM sampling demodulation. The

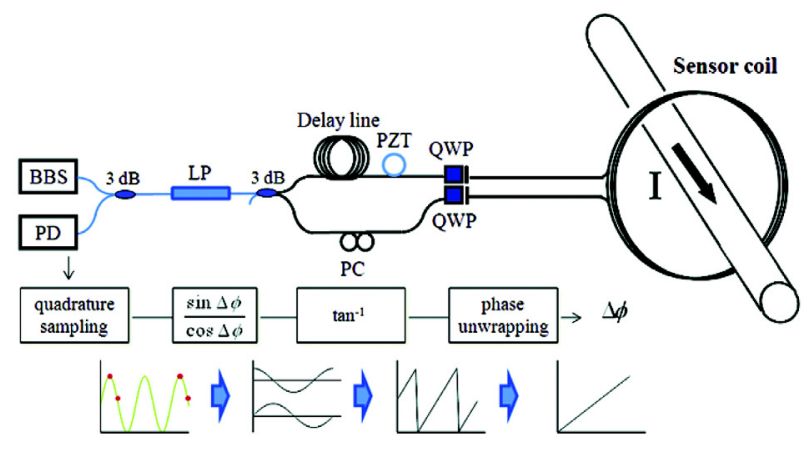

FIG. 1. The schematic diagram of the fiber-optic CT that uses a PWM sampling demodulation. (BBS: broadband source, PC: polarization controller, LP: linear polarizer, PD: photodiode, QWP: quarter-wave plate). light from a broadband source passes through a fiber-optic linear polarizer, launching the linearly polarized light to QWPs (quarter-wave-plates) of which axes are adjusted at 45 degrees with respect to the polarizer's axis. For convenience, let us assume that only the $\mathrm{x}$ component of the linear polarization passes the upper QWP, and the y component passes the lower one. Passing the QWPs, the $\mathrm{x}$ and the $\mathrm{y}$ components become the left- and the right-circular polarizations, respectively. The Faraday effect can be explained as a circular birefringence induced in the optical fiber by the magnetic field intensity. With the circular birefringence, the phase velocities of the two circularly polarized lights are different, generating a phase difference between them. The counterpropagating circularly polarized lights in the sensor coil become linearly polarized lights after passing through the QWPs again. They recombine and generate an interference signal at the photo diode of which output can be written as following equation,

$$
I_{d}=I_{0}\left\{1+\cos \left[2 \Delta \phi+\phi_{m}(t)\right]\right\}
$$

, where $I_{0}$ is the DC intensity, $\Delta \phi$ is the current-induced Faraday rotation angle and $\phi_{m}(t)$ is the phase generated by the PZT (piezoelectric transducer) phase modulator. In PWM sampling, to extract $\Delta \phi$, a sinusoidal voltage is applied to the phase modulator, generating carrier frequency in the interference signal. In principle, it is better to use a ramp modulation. Due to the mechanical resonance characteristics of the fiber stretcher, however, a sinusoidal (sometimes triangular in relatively low-speed applications) modulation is used in practice, generating a Bessel function interference signal.

In the absence of electric current, the ac-coupled PD output will be an AC signal with a fixed phase and amplitude as in figure 2(a). When a $60 \mathrm{~Hz}$ electric current is applied, the current-induced phase will shift the phase and change the amplitude of the AC signal as in figure 2(b).

To extract the current-induced phase, the interference signal is sampled twice during one period of PWM pulse as
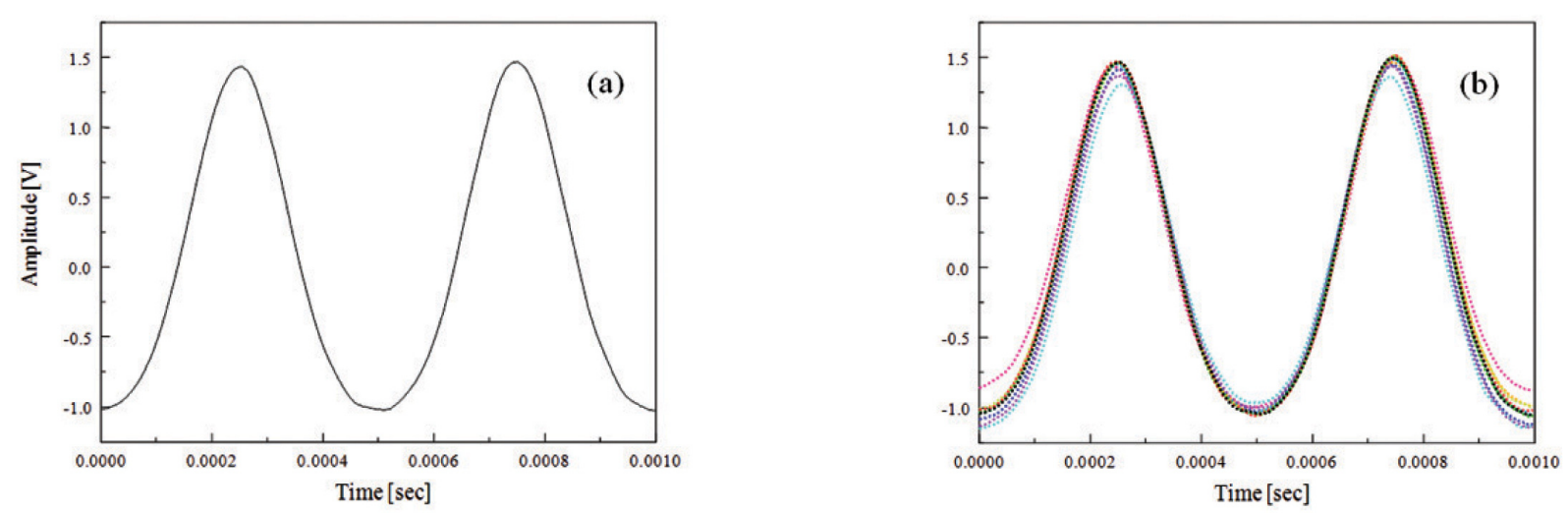

FIG. 2. PD signal with (a) no current, (b) $60 \mathrm{~Hz}$ AC current. 


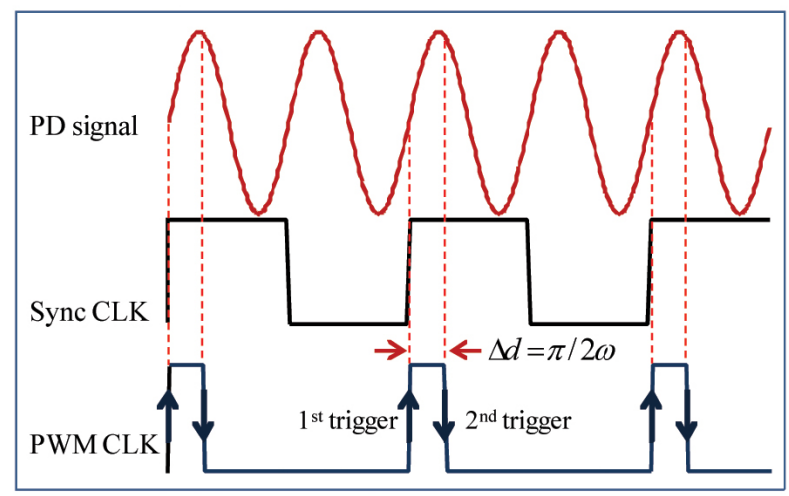

FIG. 3. Timing chart of the PWM quadrature sampling.

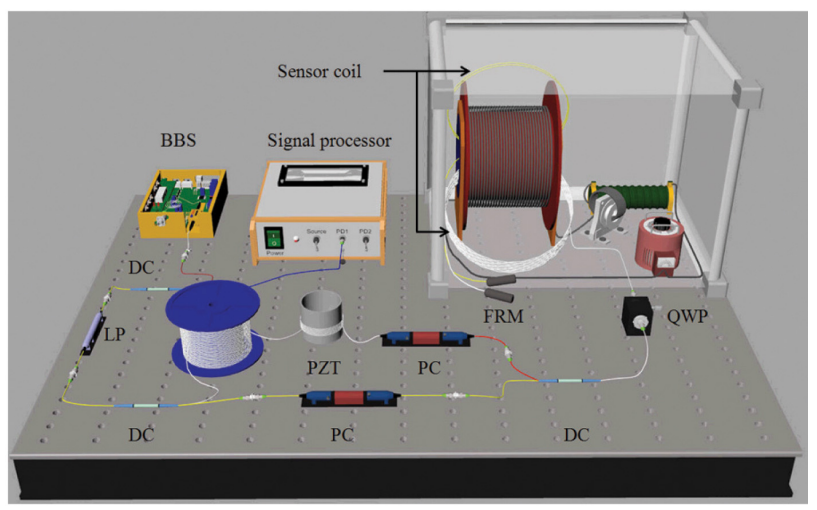

FIG. 4. Experimental setup of a fiber-optic CT that uses a PWM sampling (BBS: broadband source, PC: polarization controller, LP: linear polarizer, PD: photodiode, QWP: quarter-wave plate).

shown in figure 3 . The first sampling occurs at the rising edge of the PWM trigger, and the second sampling occurs at the falling edges of the trigger after a pulse width $\Delta d$, which generates 90-degree phase differences between the two samples. The first sampled data stream is divided by the second one. Because the data streams have 90-degree phase difference, the division generates tangent values of the current-induced phase. An arctangent function and a phase unwrapping algorithm are applied to extract the phase and to compensate for phase jumps, respectively.

\section{EXPERIMENTAL RESULTS}

Figure 4 shows the experimental setup of the suggested fiber-optic CT. As a light source, we used an EDF (erbiumdoped fiber) ASE (amplified spontaneous emission) broadband source (center wavelength: $1567 \mathrm{~nm}, 3-\mathrm{dB}$ bandwidth: $70 \mathrm{~nm}$ ) and a fiber-optic linear polarizer. We placed a cylindrical PZT fiber stretcher and a $1 \mathrm{~km}$ long delay line in one of the Mach-Zehnder interferometer arms for asymmetric phase modulation to the counter-propagating lights. A sinusoidal driving voltage $(1 \mathrm{kHz}, 12 \mathrm{Vp}-\mathrm{p})$ was given to the fiber

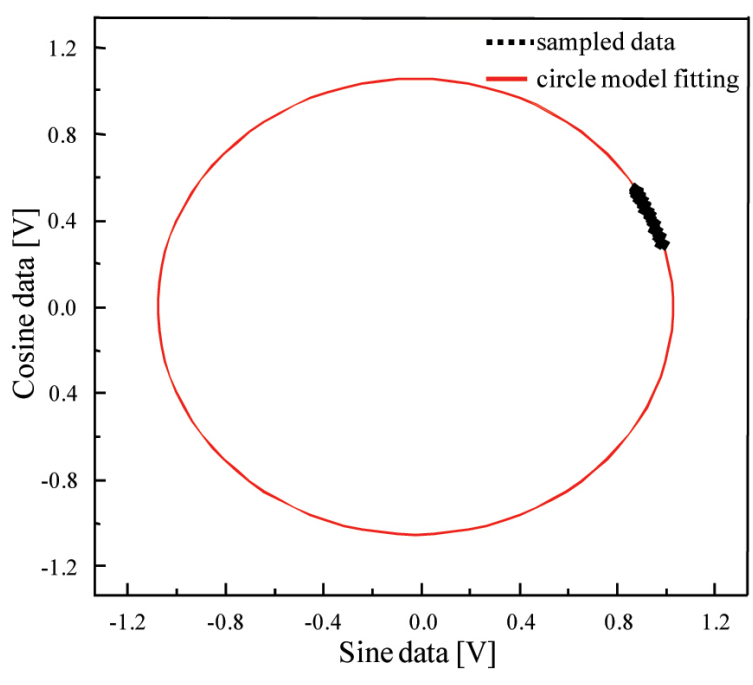

FIG. 5. Lissajous plots of sampled data streams.

stretcher to generate a phase excursion more than 90 degrees. The sensor coil we used in the experiment is a little different from that in figure 1 . It is a reflection type with a mirror attached to the end of it. It is almost the same with the in-line Sagnac interferometer in Ref. [7]. However, the working mechanism is almost the same with the Sagnac interferometer in figure 1.

To evaluate the CT system, we constructed a test current source by winding 1000 turns of copper wire, which generated up to $3550 \mathrm{AT}$ at $60 \mathrm{~Hz}$. With the maximum current output, the interference signal was PWM sampled.

Figure 5 is the Lissajous graph of the two sampled data streams. If the Lissajous plot makes a circle, then the phase difference between the sampled data is $90^{\circ}$, which is the prerequisite condition for accurate arctangent demodulation. In the experiment, because the current-induced phase change was in a limited range, the Lissajous plot was curve-fitted by a circle model, and the MSE (mean squared error) was calculated between the fitted circle and the sampled values. In the full range of input current, the MSE was less than $2.87 \times 10^{-5}$ and the corresponding phase error was calculated to be $2.35 \mathrm{mrad}$ ( $\sim 2 \%$ current measurement accuracy).

Figure 6 shows the measured profiles with increasing 60 $\mathrm{Hz}$ electric current. The reconstructed $60 \mathrm{~Hz} \mathrm{AC}$ current is apparent in figure 6. To compare the linear birefringence effects and sensitivity, we used different sensor coils: a 10-turn standard single-mode fiber and a 2-turn flint glass fiber with different types of mirror attached. Figure 7 shows the measured RMS current value of the two sensor coils with different mirrors. The flint glass fiber (Hoya Corp.), which is a lead-doped optical fiber developed to measure a high current over $180 \mathrm{kA}$, shows larger outputs although the number of turns is just one-fifth of the standard fiber coil. That could be attributed to the low birefringence and the high Verdet constant $\left(3.75 \times 10^{-6}[\mathrm{rad} / \mathrm{AT}]\right)$ of the flint glass fiber [13].

The sensitivities of the coils with a FRM were about 20\% 


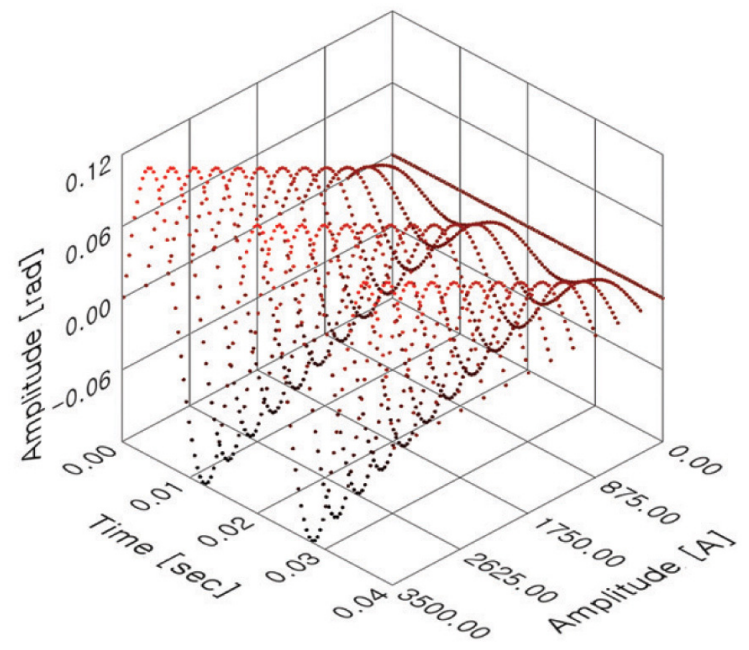

FIG. 6. Demodulated AC current outputs.

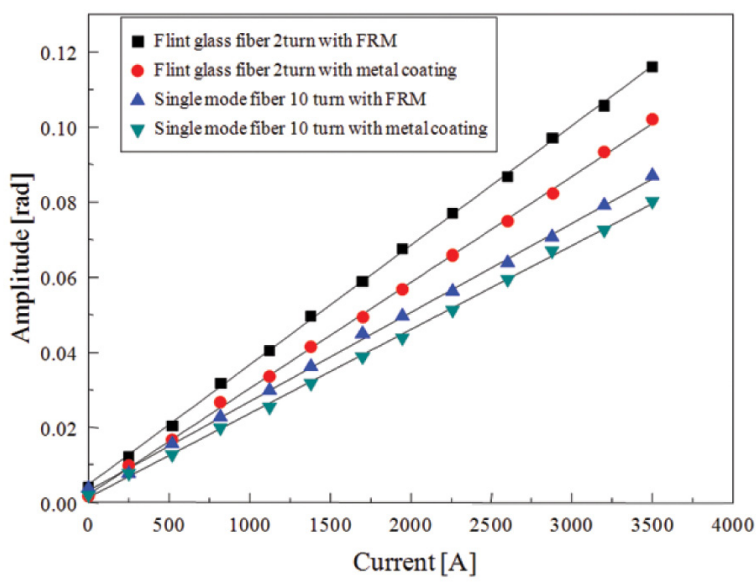

FIG. 7. Current measurement with different sensor coils.

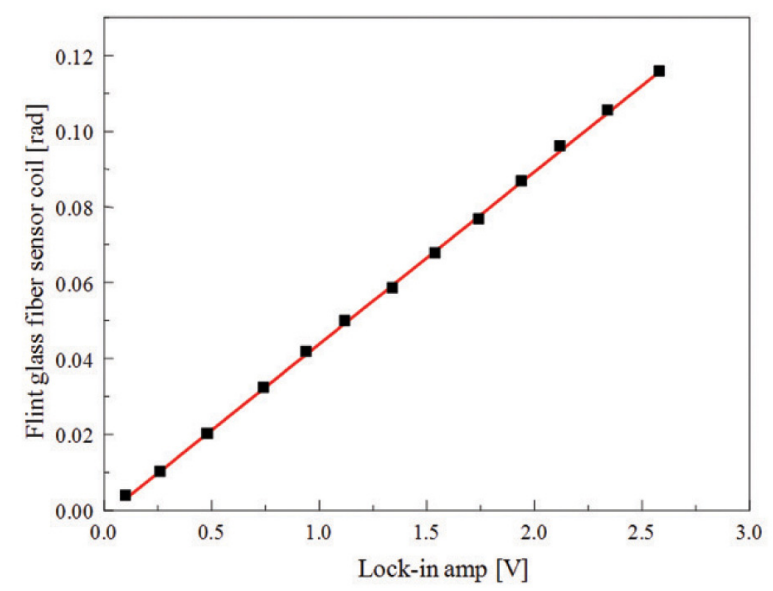

FIG. 8. Comparison of different phase demodulations: lock-in amp vs. PWM sampling

larger than those of the metal-coated coils. We used a Halleffect $\mathrm{CT}$ as a reference current sensor, and the linearity errors with the Hall-effect CT were less than $0.18 \%$, regardless of sensor coils and mirror types, over the whole measurement range. To verify the accuracy of the proposed demodulation, we measured the electric current in the same condition with a lock-in amplifier demodulation. The interference signal of the fiber-optic CT is divided by a $1 \times 2 \mathrm{BNC}$ connector and connected to a lock-in amplifier demodulation at the same time. The comparison results of current measurement are plotted in figure 8 . The two results with different demodulation techniques showed only $0.36 \%$ difference.

\section{CONCLUSION}

We constructed a fiber-optic interferometric CT using a PWM sampling demodulation. To verify the feasibility of the proposed technique, we compared the measurement result with that of a lock-in amplifier demodulation. The linearity error between the two demodulations was less than $\sim 0.36 \%$ in the input current range of $0 \sim 3550$ AT. From the experiments with different sensor coils and different mirrors, the sensitivity of a flint glass fiber with a FRM was about 3 $\mathrm{dB}$ larger than that of a standard single-mode fiber coil with a metal-coated fiber end. Because of the simplicity, the PWM sampling would be a very cost-effective demodulation technique for commercial fiber-optic CTs that require moderate measurement resolution for over-current protection uses.

\section{ACKNOWLEDGMENT}

This work was supported by the Korea Research Foundation Grant funded by the Korean Government (MOEHRD) (KRF-2007-1-D00200)

\section{REFERENCES}

1. A. Papp and H. Harms, "Magneto optical current transformer. 1: principles," Appl. Opt. 19, $3729-3734$ (1980).

2. A. J. Rogers, "Optical-fiber current measurement," Int. J. Optoelectron. 3, 391-407 (1988).

3. J. Barlow and D. N. Payne, "Production of single mode fibers with negligible intrinsic birefringence," Electron. Lett. 17, 725-726 (1986).

4. G. W. Day, K. B. Rochford, and A. H. Rose, "Fundamentals and problems of fiber current sensors," in Proc. 11th OFS Conference (Sapporo, Japan, May 1996), paper We1S1.

5. K. Bohnert, P. Gabus, J. Nehring, and H. Brandle, "Temperature and vibration insensitive fiber-optic current sensor," IEEE J. Lightwave Technol. Lett. 20, 267-276 (2002).

6. J. Blake, P. Tantaswadi, and R. T. DeCarvalho, "In-line Sagnac interferometer current sensor," IEEE Trans. Power Deliv. 11, 116-121 (1996).

7. J. Blake, "Fiber optic current sensor calibration," in Proc. 
IEEE T\&D Conference (Atlanta World Congress Center, USA, Oct. 2001), pp. 127-130.

8. Y. L. Lo, "In-fiber Bragg grating sensors using interferometric interrogations for passive quadrature signal processing," IEEE Photon. Technol. Lett. 10, 1003-1005 (1998).

9. M. O. Jeong, N. Kim, and J. H. Park, "Elemental image synthesis for integral imaging using phase-shifting digital holography," J. Opt. Soc. Korea 12, 275-280 (2008).

10. S. H. Jeon and S. K. Gil, "Measurement of a mirror surface topography using 2-frame phase-shifting digital interferometry,"
J. Opt. Soc. Korea 13, 245-250 (2009).

11. M. Song, S. Yin, and P. B. Ruffin, "Fiber Bragg grating strain sensor demodulation with quadrature sampling of a Mach-Zehnder interferometer," Appl. Opt. 39, 1106-1111 (2000).

12. J. Zhongxie and M. Song, "Fiber grating sensor array interrogation with time-delayed sampling of a wavelength-scanned fiber laser," IEEE Photon. Technol. Lett. 16, 1924-1926 (2004).

13. K. Kurosawa, "Optical current transducers using flint glass fiber as the faraday sensor element," Opt. Rev. 4, 38-44 (1997) 